Voix et Images

volxetimages

\title{
Parlez-nous d'amour ou qui parle de quoi à qui?
}

\section{Gilles Thérien}

Volume 2, numéro 2, décembre 1976

Paul Chamberland

URI : https://id.erudit.org/iderudit/200062ar

DOI : https://doi.org/10.7202/200062ar

Aller au sommaire du numéro

Éditeur(s)

Les Presses de l'Université du Québec

ISSN

0318-9201 (imprimé)

1705-933X (numérique)

Découvrir la revue

Citer cet article

Thérien, G. (1976). Parlez-nous d'amour ou qui parle de quoi à qui? Voix et Images, 2(2), 286-290. https://doi.org/10.7202/200062ar d'utilisation que vous pouvez consulter en ligne.

https://apropos.erudit.org/fr/usagers/politique-dutilisation/ 


\section{Parlez-nous d'amour ou qui parle de quoi à qui?}

\section{Première réflexion}

Celui qui veut aller au cinéma doit choisir dans la section ad hoc d'un journal quelconque le film qu'il verra. La publicité est là pour lui arracher un consentement. Examinons celle de Parlez-nous d'amour:

(en lettres super-majuscules)

UN FILM CHOC / UN ÉVÉNEMENT / UN GRAND SUCCES !

(en letțres normales)

1. «Une bombe... Une date dans l'histoire de notre cinéma... Lord fait éclater le "Watergate» du monde des variétés. C'est le film le plus important de l'année. " Journal de Montréal

2. “Un film-vérité, une réussite où le rythme est intéressant et soutenu. Quand on dit que la réalité dépasse la fiction, rien n'est plus vrai." Montréal-Matin

3. "Un film à voir. " CKMF

4. "Un excellent film, avec un sujet bien traité et un Jacques Boulanger impeccable. Ne le manquez pas. " Radio-Canada

5. «Un film qui va faire parler... énormément. "Télé-Métropole

6. "Un film incommensurablement choquant... Les comédiens jouent tous merveilleusement bien. Un des "meilleurs" films de Jean-Claude Lord. " CJMS

7. "Merveilleusement bien joué, un très beau film. " CFGL

8. "Une critique impitoyable, un film qui donne à voir, une direction d'acteurs remarquable. "Cinéma-Québec

9. "Jean-Claude Lord a réussi un coup de maître et ce film va obtenir un succès fulgurant. "Vedettes québécoises 
Le premier compte est simple à faire: deux quotidiens, un hebdomadaire, une revue, une station de télévision, quatre stations de radio. Couverture complète en somme du côté des média mais aussi message acheminé à un très fort pourcentage de la population de la région de Montréal, sans aucune exclusion d'une minorité comme les intellectuels par exemple. Quelle est donc la qualité de ce message? II est contenu tout entier en 141 mots. Une analyse de l'utilisation des mots en rapport avec leur fréquence d'utilisation dans la langue ${ }^{1}$ permet de se faire une meilleure idée du niveau du message:

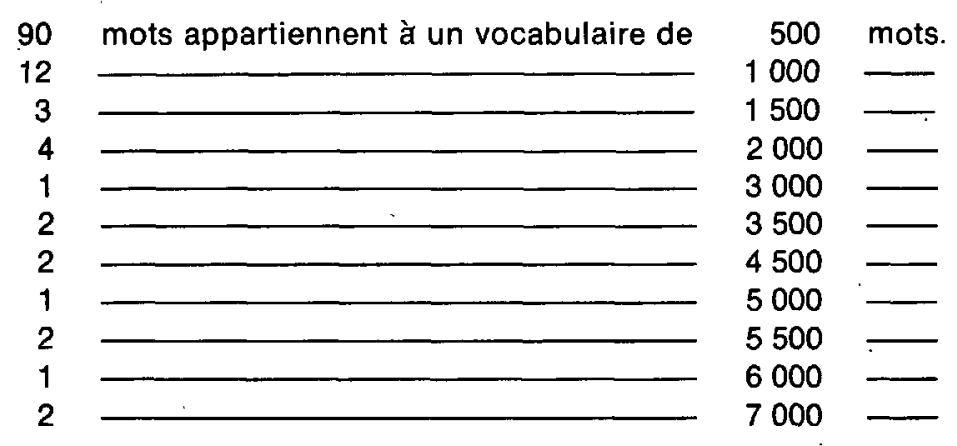

21 mots sont au-delà de ces catégories. Le mot «film» est répété 11 fois et dans le contexte, on pourrait l'assimiler à un vocabulaire de 500 mots. On compte aussi 5 noms propres, ce qui laisse 5 mots appartenant à un vocabulaire plus étendu. Ces mots sont: fiction, impeccable, incommensurablement, impitoyable, fulgurant. Il s'agit d'un nom, de trois adjectifs et d'un adverbe emphatique. Appartiennent à des classes voisines, bombe, cinéma, choquant (choc), merveilleusement, comédiens, rythme. La première hypothèse est que ce message est pour le moins économique sur le plan culturel. II ne dit que les choses que tout le monde peut comprendre. Mais encore, que dit-il?

Le message lui-même ne repose que sur 58 mots différents, le reste n'étant constitué que d'articulations grammaticales indispensables: articles, verbes (copule et auxiliaires), conjonctions, relatifs. Les principaux mots se laissent rassembler en cinq classes dont nous donnerons les principaux éléments :

I. Film-bombe-vérité-choc-critique impitoyable

II. Date-événement-film de l'année-histoire de notre cinéma

III. Bien joué-bien dirigé-sujet bien traité-rythme intéressant et soutenucoup de maître

IV. Succès fulgurant-merveilleusement-remarquable-beau-meilleur

V. Jean-Claude Lord-Jacques Boulanger-comédiens 
La comparaison de ces classes entre elles ne peut que confirmer l'inexistence de l'information, puisque, comme nous pouvons le voir, les éléments constitutifs de chacune des classes ont une tendance à être redondants. Toutefois, l'analyse de la première citation pourrait servir de modèle d'information. En l'examinant nous y trouverons un tissu allusif qui ne manque pas d'intérêt. La citation comporte quatre phrases qu'on peut disposer selon trois rubriques fort simples: sujet, verbe et complément.

\section{SUJET}

1. Bombe

2. Date

3. Lord
VERBE

(sous-entendu)

(sous-entendu)

fait éclater

4. Le film le plus im-

est

COMPLÉMENT portant de l'année

On ne peut faire autrement que noter les associations possibles: bombefait éclater, date-film de l'année, film le plus important-Lord. Or si on rajoute à ces informations celles contenues dans les autres citations, on retrouve ce réseau allusif organisé autour de Lord, de son cinéma et du monde des variétés:

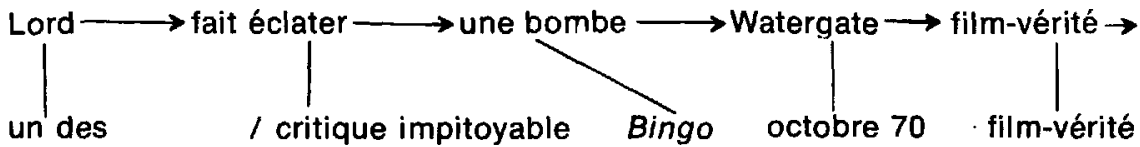

meilleurs films

monde des variétés $\rightarrow$ comédiens/population $\rightarrow$ marionnette du spectacle<smiles>CCCC[Te]C</smiles>

monde de la politique

politiciens/population

manipulateur-traître

En somme, la publicité écrite nous promet un film d'action, la révélation et la dénonciation d'un autre complot (inattendu, bien sûr!) et ce, de façon encore supérieure (ou au moins égale) à son film précédent, Bingo.

\section{Seconde réflexion}

Puisqu'on y est, pourquoi ne pas parler de l'affiche publicitaire? L'importance est accordée à une tête de femme coupée en deux. Un homme, petit, se tient dans un équilibre difficile sur les deux morceaux de la 
tête à la fois. II est baigné d'un faisceau lumineux issu d'un réflecteur situé dans le cadre gauche de l'affiche. Au-dessus de ce réflecteur, une caméra de télévision, visant hors-cadre, fait l'effet d'un ornement. Au bas de la tête de la jeune fille, une chaise roulante et un corps de femme, apparemment étendu sans vie. Plus bas, nous trouvons titre et générique.

Voilà donc les éléments du complot. Les éléments principaux, en fonction de leur importance graphique, sont certainement cette tête de fille littéralement sciée en deux mais qui n'en éprouve qu'une souffrance modérée: deux larmes sous l'œil gauche. Les deux autres personnages sont peu caractérisés: un chanteur, puisqu'il tient un micro qui pend au bout d'un fil et oscille comme un pendule, et ce cadavre de femme, relié par contiguïté à la chaise roulante. Le graphisme est loin d'être simple. II se présente comme un rébus et ne résume nullement le propos du film. II ne fait qu'entretenir l'ambiguïté du message publicitaire en évoquant un amour brisé et de la violence, tout juste ce qu'il faut pour donner un air de vraisemblance au titre Parlez-nous d'amour. Malheureusement, le sujet est moins violent, plus vulgaire, sans grande profondeur et ne fait que nous raconter une chose que l'on savait déjà : le talent passe par le c..., pour les producteurs comme pour les spectateurs.

\section{Troisième réflexion}

J'ai payé $\$ 3.50$ pour voir un film qui en vaut environ $\$ 450000$. Or pour récupérer la mise de fonds, il faut aller chercher un minimum de $\$ 2200000$. au guichet, c'est-à-dire plus ou moins un million de spectateurs. Et le surplus sera pour le «fulgurant». Bonne chance!

\section{Quatrième et dernière réflexion}

Tout ce qui a été dit précédemment n'a que deux justifications: le malaise et le fait que la publicité est venue faire ce que le film dénonce mal.

II serait d'ailleurs temps de parler du film lui-même qui commence par un inter-titre «gros gros merci à l'équipe» et puis, pourquoi pas? bonjour à papa et à maman, de même qu'à tous les bons amis du monde des variétés sans lesquels ce film n'aurait pas été possible. On se croirait à un match de lutte télévisé il y a quinze ans. L'analogie se poursuit quand, dans un second inter-titre, Jean-Claude Lord et Michel Tremblay nous préviennent de l'authenticité des faits que nous allons voir. II ne s'agit pas, paraît-il, de l'imagination débordante d'un auteur. Merveilleux lapsus du singulier du mot “auteur». Le film est signé Tremblay. On y retrouve son amour exagéré des femmes, sa tendresse pour elles, sa fierté d'être québécois, son respect tout créditiste de la personne humaine, son raffine- 
ment, son langage à la Pierre Beaudry, ses personnages d'une profondeur abyssale, son sens de l'humour, son bon goût universel, sa construction, habilement dialectique, des rapports entre les personnages avec ce qu'il faut de tension dramatique, de rebondissement inattendu, sa finesse d'observation du genre humain québécois qui n'est pas loin de le consacrer re-Balzac. La veine de Tremblay n'étant ni épuisée ni épuisable, il·n'est pas étonnant de voir que le film aurait pu être fait il y a cinq ans, dix ans, ou même... mais il n'était pas né!

Lord, lui, a consciencieusement dit "Silence, on tourne/ Action / Stop". Il a non moins consciencieusement collé bout à bout les morceaux d'une honnête émission de télévision. D'imagination créatrice, point; de direction d'acteurs, point. Boulanger a fait ce qu'il fait tous les jours, ni plus ni moins. Quant aux autres... il est difficile de diriger des caricatures.

Le film qui raconte les déboires d'un animateur de télévision qui se met à penser (un peu) et l'univers sordide du monde des variétés, utilise exactement les mêmes procédés qu'il semble vouloir dénoncer: vedette phallocratique à peine dénudée, producteurs véreux et vicieux, publiç laid et bête, morale qui ne peut que conduire à la dépression. $\mathrm{Ce}$ film «populaire» est un monument de vulgarité (vulgus). Ce sont des films du genre (Canal 10 et moins...) qui vous font parfois regretter de ne pas être né en Papouasie. II faudra un jour avoir le courage de dénoncer nos pseudo-créateurs, qui tirent leurs cachets de ceux qu'ils critiquent gentiment en prenant bien soin de ne conscientiser personne, à condition de ne pas tomber dans le lyrisme lemelinien, autre genre de vulgarité.

Ah oui, j'oubliais: la publicité la plus éclairante $\left(n^{\circ} 1\right)$ appartient au monde des variétés littéraires de Péladeau, dont un des passe-temps favoris est la philosophie.

Sainte Zazie, priez pour nous, pauvres Québécois!

Gilles Thérien

1. Cf. Word Frequency Dictionary, compilé par H.S. Eaton, Dover, New York, 1960. Ce dictionnaire est quadrilingue. 